Bulletin of the Institute of Mathematics

Academia Sinica (New Series)

Vol. 13 (2018), No. 2, pp. 227-247

DOI: 10.21915 /BIMAS.2018203

\title{
THE SYLOW SUBGROUPS OF A FINITE REDUCTIVE GROUP
}

\author{
MICHEL ENGUEHARD ${ }^{1, a}$ AND JEAN MICHEL ${ }^{2, b}$ \\ Dedicated to professor George Lusztig on the occasion of his 70th birthday \\ ${ }^{1}$ UFR de Mathématiques, Université Denis Diderot - Paris 7, Bat. Sophie Germain Case 7012, 75205 \\ Paris Cedex 13, France. \\ ${ }^{a}$ E-mail: michel.enguehard@imj-prg.fr \\ ${ }^{2}$ UFR de Mathématiques, Université Denis Diderot - Paris 7, Bat. Sophie Germain Case 7012, 75205 \\ Paris Cedex 13, France. \\ ${ }^{b}$ E-mail: jean.michel@imj-prg.fr
}

\|\|

\section{Abstract}

We describe the structure of Sylow $\ell$-subgroups of a finite reductive group $\mathbf{G}\left(\mathbb{F}_{q}\right)$ when $q \not \equiv 0(\bmod \ell)$ that we find governed by a complex reflection group attached to $\mathbf{G}$ and $\ell$, which depends on $\ell$ only through the set of cyclotomic factors of the generic order of $\mathbf{G}\left(\mathbb{F}_{q}\right)$ whose value at $q$ is divisible by $\ell$. We also tackle the more general case of groups $\mathbf{G}^{F}$ where $F$ is an isogeny some power of which is a Frobenius morphism.

\section{Introduction}

Definition 1.1. Let $\mathbf{G}$ be a connected reductive group over $\overline{\mathbb{F}}_{p}$, and $F$ an isogeny such that some power of $F$ is a Frobenius endomorphism; then $\mathbf{G}^{F}$ is what we call a finite reductive group. To this situation we attach a positive real number $q$ such that for some integer $n$, the isogeny $F^{n}$ is the Frobenius endomorphism attached to a $\mathbb{F}_{q^{n-s t r u c t u r e . ~}}$

The goal of this note is to describe the Sylow $\ell$-subgroups of $\mathbf{G}^{F}$ when $\ell$ is a prime different from $p$ and $\mathbf{G}$ is semisimple. The structure of the Sylow $\ell$-subgroups of a Chevalley group was first described by [6] where they observed that they had a large normal abelian subgroup $(\mathbb{Z} / n)_{\ell}^{a}$ where

Received May 3, 2016 and in revised form July 22, 2016.

AMS Subject Classification: 20G40, 20D20.

Key words and phrases: Reductive groups, Sylow subgroups. 
$n$ is the $\ell$-part of $\Phi_{d}(q)$, where $d$ is the multiplicative order of $q(\bmod \ell)$, and they computed $a$ case by case.

In 1992 [3] exhibited subtori of $\mathbf{G}^{F}$ attached to eigenspaces of elements of the Weyl reflection coset of $(\mathbf{G}, F)$ whose $F$-stable points are the large abelian groups of [6]. To these eigenspaces are attached complex reflection groups by Springer's theory.

We show that the structure of the Sylow $\ell$-subgroups of $\mathbf{G}^{F}$ is determined by these complex reflection groups. The results of this note in the case when $F$ is a Frobenius were obtained by the first author in an unpublished note [5] of 1992; the second author has found a simpler (containing more casefree steps) proof which is an occasion to publish these results. Some of our results appeared also implicitly in [7].

The second author wishes to thank Carles Broto for a visit to Barcelona, which started him thinking about this topic.

We thank Raphaël Rouquier for discussions which helped with the proofs of Propositions 2.8 and 2.17(4).

\section{The Generic Sylow Theorems}

Let $\mathbf{G}$ be as in Definition 1.1; an $F$-stable maximal torus $\mathbf{T}$ of $\mathbf{G}$ defines the Weyl group $W=N_{\mathbf{G}}(\mathbf{T}) / \mathbf{T}$, that we may identify to a reflection subgroup of $G L(X(\mathbf{T}))$ where $X(\mathbf{T}):=\operatorname{Hom}\left(\mathbf{T}, \mathbb{G}_{m}\right)$, attached to the root system $\Sigma \subset X(\mathbf{T})$ of $\mathbf{G}$ with respect to $\mathbf{T}$. The isogeny $F$ induces a $p$-morphism $F^{*} \in \operatorname{End}(X(\mathbf{T}))$ by the formula $F^{*}(x)=x \circ F$ for $x \in X(\mathbf{T})$, that is there is a permutation $\sigma$ of $\Sigma$ such that for $\alpha \in \Sigma$ we have $F^{*}(\alpha)=q_{\alpha} \sigma(\alpha)$ for some power $q_{\alpha}$ of $p$; in particular $F^{*} \in N_{\operatorname{End}(X(\mathbf{T}))}(W)$.

If $q, n$ are as in Definition 1.1 then $F^{* n}$ is $q^{n}$ times an element of $G L(X(\mathbf{T}))$ of finite order, thus over $X(\mathbf{T}) \otimes \mathbb{Z}\left[q^{-1}\right]$ we have $F^{*}=q \phi$ where $\phi$ is an automorphism of finite order which normalizes $W$. We call $W \phi$ the reflection coset associated to $(\mathbf{G}, F)$.

Our setting is more general than that of [3] who considered only the special cases where $F$ is a Frobenius endomorphism, or where $\mathbf{G}^{F}$ is a Ree or Suzuki group. The results of the next subsection allow to extend the definition of Sylow $\Phi$-subtori of [3] to any $(\mathbf{G}, F)$ as in Definition 1.1. 


\section{$F$-indecomposable tori}

Definition 2.1. For $\mathbf{G}, F$ as in Definition 1.1, a non-trivial subtorus of $\mathbf{G}$ is called $F$-indecomposable if it is $F$-stable and contains no proper non-trivial $F$-stable subtorus.

We say that a group $G$ is an almost direct product of subgroups $G_{1}$ and $G_{2}$ if they commute, generate $G$ and have finite intersection, and we define similarly an almost direct product of $k$ subgroups by induction on $k$.

Proposition 2.2. For $\mathbf{G}, F$ as in Definition 1.1 , any F-stable subtorus $\mathbf{T}$ of $\mathbf{G}$ is an almost direct product of $F$-indecomposable tori $\mathbf{S}_{1}, \ldots, \mathbf{S}_{k}$ and $\left|\mathbf{T}^{F}\right|=\left|\mathbf{S}_{1}^{F}\right| \ldots\left|\mathbf{S}_{k}^{F}\right|$.

Proof. An $F$-stable subtorus $\mathbf{S}$ corresponds to a pure $F$-stable sublattice $X^{\prime} \subset X:=X(\mathbf{T})$ (see for example [1, III, Proposition 8.12]). Let $d$ be the smallest power of $F$ which is a split Frobenius, thus on $X(\mathbf{T})$ we have $F^{* d}=$ $q^{d} I d$. Let $\pi \in \operatorname{End}(X \otimes \mathbb{Q})$ be a projector on $X^{\prime} \otimes \mathbb{Q}$. Then in $\operatorname{End}(X \otimes \mathbb{Q})$ we can define the $F$-invariant projector $\pi^{\prime}:=d^{-1} \sum_{i=1}^{d} F^{* i} \pi F^{*-i}$ and $\operatorname{Ker} \pi^{\prime} \cap X$ is another $F$-stable pure sublattice which after tensoring by $\mathbb{Q}$ becomes a complement to $X^{\prime} \otimes \mathbb{Q}$. This corresponds to an $F$-stable subtorus $\mathbf{S}^{\prime}$ such that $K:=\mathbf{S} \cap \mathbf{S}^{\prime}$ is finite and $\mathbf{T}=\mathbf{S S}^{\prime}$. Iterating, we get the first part of the proposition.

The second part of the proposition results from the next two lemmas.

Lemma 2.3. For $\mathbf{G}, F$ as in Definition 1.1 , and $K$ an $F$-stable finite normal subgroup of $\mathbf{G}$, then $\left|(\mathbf{G} / K)^{F}\right|=\left|\mathbf{G}^{F}\right|$.

Proof. First, we notice that $K$ is central, thus abelian, since conjugating by $\mathbf{G}$ being continuous must be trivial on $K$.

Then, the Galois cohomology long exact sequence: $1 \rightarrow K^{F} \rightarrow \mathbf{G}^{F} \rightarrow$ $(\mathbf{G} / K)^{F} \rightarrow H^{1}(F, K) \rightarrow 1$ shows the result using that $\left|K^{F}\right|=\left|H^{1}(F, K)\right|$.

Lemma 2.4. Let $\mathbf{G}$ as Definition 1.1 be an almost direct product of F-stable connected subgroups $\mathbf{G}=\mathbf{G}_{1} \ldots \mathbf{G}_{k}$. Then $\left|\mathbf{G}^{F}\right|=\left|\mathbf{G}_{1}^{F}\right| \ldots\left|\mathbf{G}_{k}^{F}\right|$.

Proof. It is enough to consider the case $k=2$ and then iterate. Thus, we assume $\mathbf{G}=\mathbf{G}_{1} \mathbf{G}_{2}$ where $K=\mathbf{G}_{1} \cap \mathbf{G}_{2}$ is finite. We quotient by $K$, which makes the product direct, and apply Lemma 2.3 twice. 
Lemma 2.5. Let $\mathbf{S}$ be an F-indecomposable torus, let $\eta$ be the smallest power such that $q^{\eta} \in \mathbb{Z}$, and let $d$ be the smallest power such that $F^{d \eta}$ is a split Frobenius on $\mathbf{S}$. Let $F^{*}=q \phi$ on $X(\mathbf{S})$; then the characteristic polynomial $\Phi$ of $\phi$ is a factor in $\mathbb{Z}\left[x, q^{-1}\right]$ of $\Phi_{d}\left(x^{\eta}\right)$, where $\Phi_{d}(x)$ denotes the d-th cyclotomic polynomial. Further $q^{\operatorname{deg} \Phi} \Phi(x / q) \in \mathbb{Z}[x]$ is irreducible and $\left|\mathbf{S}^{F}\right|=\Phi(q)$.

Proof. Since $F^{* d \eta}$ acts as $q^{d \eta}$ on $X:=X(\mathbf{S})$, the minimal polynomial $P$ of $F^{*}$ divides $x^{d \eta}-q^{d \eta}$.

The polynomial $P$ is irreducible over $\mathbb{Z}$, otherwise a proper nontrivial factor $P_{1}$ defines an $F^{*}$-stable pure proper non-trivial sublattice $\operatorname{Ker}\left(P_{1}\left(F^{*}\right)\right)$ of $X$, which contradicts $F$-indecomposability of $\mathbf{S}$.

It follows that $X$ is a $\mathbb{Z}[x] / P$-module by making $x$ act by $F^{*}$, and $X \otimes \mathbb{Q}[x] / P$ is a one-dimensional $\mathbb{Q}[x] / P$-vector space, otherwise a proper nontrivial subspace would define an $F^{*}$-stable pure sublattice of $X$. It follows that $\operatorname{dim} \mathbf{S}=\operatorname{deg} P=\operatorname{dim} X$ and thus $P$ is also the characteristic polynomial of $F^{*}$.

We have in $\mathbb{Z}[x]$ the equality $x^{d \eta}-q^{d \eta}=\prod_{d^{\prime} \mid d}\left(q^{\eta \operatorname{deg} \Phi_{d^{\prime}}} \Phi_{d^{\prime}}\left(x^{\eta} / q^{\eta}\right)\right)$. Since $P$ is irreducible it divides one of the factors, and since $d \eta$ is minimal such that $F^{* d \eta}=q^{d \eta} I d$, that is minimal such that $P$ divides $x^{d \eta}-q^{d \eta}$, we have that $P$ divides $q^{\eta \operatorname{deg} \Phi_{d}} \Phi_{d}\left(x^{\eta} / q^{\eta}\right)$; equivalently $\Phi=q^{-\operatorname{deg} P} P(q x)$ divides $\Phi_{d}\left(x^{\eta}\right)$.

We have $\left|\mathbf{S}^{F}\right|=\left|\operatorname{Irr}\left(\mathbf{S}^{F}\right)\right|=\left|X /\left(F^{*}-1\right) X\right|=\operatorname{det}\left(F^{*}-1\right)=(-1)^{\operatorname{deg} P} P(1)$ $=(-q)^{\operatorname{deg} \Phi} \Phi(1 / q)$ where the second equality reflects the well known group isomorphism $\operatorname{Irr}\left(\mathbf{S}^{F}\right) \simeq X /\left(F^{*}-1\right) X$ and the third is a general property of lattices. Finally, since $\Phi$ is real and divides $\Phi_{d}\left(x^{\eta}\right)$, its roots are stable under taking inverses, thus $(-q)^{\operatorname{deg} \Phi} \Phi(1 / q)=\Phi(q)$.

We call $q$-cyclotomic the polynomials $\Phi$ of Lemma 2.5. In other terms

Definition 2.6. For $q$ as in Definition 1.1, where $q^{\eta}$ is the smallest power of $q$ in $\mathbb{Z}$, we call $q$-cyclotomic the monic polynomials $\Phi \in \mathbb{Z}\left[x, q^{-1}\right]$ such that $q^{\operatorname{deg} \Phi} \Phi(x / q)$ is a $\mathbb{Z}[x]$-irreducible factor of some $x^{d \eta}-q^{d \eta}$.

In the study of semisimple reductive groups we will need the $q$-cyclotomic polynomials of Lemma 2.7. Note that if $d$ is minimal in Definition 2.6, then 
$\Phi$ is a factor in $\mathbb{Z}\left[x, q^{-1}\right]$ of $\Phi_{d}\left(x^{\eta}\right)$. We are interested in that number $d$ rather than $d \eta$, and to emphasize this we write $\Phi_{\eta, d}$ in the following examples.

Lemma 2.7. When $q \in \mathbb{Z}$, the $q$-cyclotomic polynomials are the cyclotomic polynomials.

When $q$ is an odd power of $\sqrt{2}$, the following polynomials are $q$-cyclotomic: $\Phi_{2,1}(x):=\Phi_{1}\left(x^{2}\right), \Phi_{2,2}(x):=\Phi_{2}\left(x^{2}\right), \Phi_{2,6}(x):=\Phi_{6}\left(x^{2}\right)$, the factors $\Phi_{2,4}^{\prime}:=$ $x^{2}+\sqrt{2} x+1$ and $\Phi_{2,4}^{\prime \prime}:=x^{2}-\sqrt{2} x+1$ of $\Phi_{4}\left(x^{2}\right)$, and the factors $\Phi_{2,12}^{\prime}:=$ $x^{4}+x^{3} \sqrt{2}+x^{2}+x \sqrt{2}+1$ and $\Phi_{2,12}^{\prime \prime}:=x^{4}-x^{3} \sqrt{2}+x^{2}-x \sqrt{2}+1$ of $\Phi_{12}\left(x^{2}\right)$.

When $q$ is an odd power of $\sqrt{3}$, the following polynomials are $q$-cyclotomic: $\Phi_{2,1}(x), \Phi_{2,2}(x)$ and the factors $\Phi_{2,6}^{\prime}:=x^{2}+x \sqrt{3}+1$ and $\Phi_{2,6}^{\prime \prime}:=x^{2}-x \sqrt{3}+1$ of $\Phi_{6}\left(x^{2}\right)$.

Proof. When $q \in \mathbb{Z}$ the formula $P \mapsto q^{-\operatorname{deg} P} P(q x)$ establishes a bijection between $\mathbb{Z}[x]$-irreducible factors of $x^{d}-q^{d}$ and $\mathbb{Z}[x]$-irreducible factors of $x^{d}-1$, that is cyclotomic polynomials, which gives the first case of the lemma.

For the other cases, we have to check for each given $\Phi$ that $q^{\operatorname{deg} \Phi} \Phi(x / q)$ is in $\mathbb{Z}[x]$ and irreducible.

Proposition 2.8. Let $\mathbf{S}, \eta, d, \Phi$ be as in Lemma 2.5 and let $P=$ $q^{\operatorname{deg} \Phi} \Phi\left(x^{\eta} / q^{\eta}\right)$ be the characteristic polynomial of $F^{*}$.

(1) Assume that either $q \in \mathbb{Z}$ or that $\mathbb{Z}\left[x, q^{-\eta}\right] / P$ is integrally closed. Then $\mathbf{S}^{F} \simeq \mathbb{Z} / \Phi(q)$.

(2) Let $m$ be a divisor of $\Phi(q)$, and assume either that $d \in\{1,2\}$ and $q \in \mathbb{Z}$ or that $m$ is prime to $d \eta$; then we have a natural isomorphism $\operatorname{Irr}\left(\mathbf{S}^{F}\right) / m \operatorname{Irr}\left(\mathbf{S}^{F}\right) \simeq \operatorname{Ker}\left(F^{*}-1 \mid X(\mathbf{S}) / m X(\mathbf{S})\right)$.

Proof. Proceeding as in the proof of Lemma 2.5 we set $X=X(\mathbf{S})$ and $\bar{X}=X /\left(F^{*}-1\right) X \simeq \operatorname{Irr}\left(\mathbf{S}^{F}\right)$. Letting $x$ act as $F^{*}$ makes $X$ into a $\mathbb{Z}[x] / P$ module, and $\bar{X}$ a $\mathbb{Z}[x] /(P, x-1)$-module. Since $\mathbb{Z}[x] /(P, x-1)=\mathbb{Z} / P(1)=$ $\mathbb{Z} / \Phi(q)$ we find that the exponent of $\bar{X}$ divides $\Phi(q)$.

Let $A:=\mathbb{Z}\left[x, q^{-\eta}\right] / P$. The extension $\mathbb{Z}[x] / P \hookrightarrow A / P$ is flat thus $\bar{X} \otimes_{\mathbb{Z}[x] / P} A \simeq X^{\prime} /\left(F^{*}-1\right) X^{\prime}$ where $X^{\prime}=X \otimes_{\mathbb{Z}[x] / P} A$; and since the exponent of $\bar{X}$ divides $\Phi(q)$ which is prime to $q^{\eta}$, we have $\bar{X} \simeq \bar{X} \otimes_{\mathbb{Z}[x] / P} A$. Under the assumptions of (1) the $\operatorname{ring} A$ is Dedekind: if $\eta \neq 1$ then $A$ is integrally closed thus Dedekind; if $\eta=1$ then $A \simeq \mathbb{Z}\left[x, q^{-1}\right] / \Phi_{d}$ where the isomorphism 
is given by $x \mapsto x / q$, and is a localization of the Dedekind ring $\mathbb{Z}[x] / \Phi_{d}$ by $q$. Thus $X^{\prime}$ identifies to a fractional ideal $\mathfrak{I}$ of $A$ and $\bar{X} \simeq \mathfrak{I} /(x-1) \mathfrak{I}$. If $e$ is the exponent of $\bar{X}$ we have thus $e \mathfrak{I} \subset(x-1) \mathfrak{I}$, which implies that $x-1$ divides $e$ in $A$. This in turn implies that the norm $(-1)^{\operatorname{deg} P} P(1)=\Phi(q)$ of $(x-1)$ divides $e$ in $\mathbb{Z}$, thus $e=\Phi(q)$ and $\bar{X} \simeq \mathbb{Z} / \Phi(q)$ and the same isomorphism holds for the dual abelian group $\mathbf{S}^{F}$.

For (2), note that by construction $\bar{X} / m \bar{X}$ is the biggest quotient of $X$ on which both $F^{*}-1$ and the multiplication by $m$ vanish. It is thus equal to the biggest quotient of $X / m X$ on which $F^{*}-1$ vanishes. Thus the question is to see that $\operatorname{Ker}\left(F^{*}-1\right)$ has a complement in $X / m X$.

If $q \in \mathbb{Z}$ and $d \in\{1,2\}$ we have $P=x \pm q$ so $X \simeq \mathbb{Z}$ on which $F^{*}$ acts by $\mp q$ and $\bar{X}=X /(q \pm 1)$ of which $X / m X$ is a quotient, so $F^{*}-1$ vanishes on $X / m X$ which is thus equal to $\bar{X} / m \bar{X}$ and there is nothing to prove.

Assume now $m$ prime to $d \eta$. There exists $R \in \mathbb{Z}[x]$ such that in $\mathbb{Z}[x]$ we have $P=(x-1) R+P(1)$. Taking derivatives, we get $P^{\prime}=(x-1) R^{\prime}+$ $R$, whence $R(1)=P^{\prime}(1)$. Let $\delta$ be the discriminant of $P$; we can find polynomials $M, N \in \mathbb{Z}[x]$ such that $M P+N P^{\prime}=\delta$, which evaluating at 1 gives $M(1) P(1)+N(1) P^{\prime}(1)=\delta$. Since $q$ is prime to $P(1)$, thus to $m$, and $\delta$ is a divisor of the discriminant of $X^{d \eta}-q^{d \eta}$, equal to $q^{d \eta(d \eta-1)}(d \eta)^{d \eta}$, thus prime to $m$, we find that $P^{\prime}(1)$ is prime to $m$. In $(\mathbb{Z} / m)[x]$ we have $P=(x-1) R$, thus applied to $F^{*}$ we get that on $X / m X$ we have $0=$ $P\left(F^{*}\right)=\left(F^{*}-1\right) R\left(F^{*}\right)$, whence $\operatorname{Ker}\left(F^{*}-1\right)+\operatorname{Ker}\left(R\left(F^{*}\right)\right)=X / m X$. Since $R(1)$ is prime to $m$, we can write $1 \equiv Q(x-1)+a R$ in $(\mathbb{Z} / m)[x]$ for some $Q \in(\mathbb{Z} / m)[x]$ and $a$ the inverse $(\bmod m)$ of $R(1)$. This proves that $\operatorname{Ker}\left(F^{*}-1\right) \cap \operatorname{Ker}\left(R\left(F^{*}\right)\right)=0$ thus $X / m X$ is the direct sum of $\operatorname{Ker}\left(F^{*}-1\right)$ and $\operatorname{Ker}\left(R\left(F^{*}\right)\right)$.

Complex reflection cosets. (1) to (3) below are classical results of Springer and Lehrer.

Proposition 2.9. Let $V$ be a finite dimensional vector space over a subfield $k$ of $\mathbb{C}$, let $W \subset G L(V)$ be a finite complex reflection group and let $\phi \in$ $N_{G L(V)}(W)$, so that $W \phi$ is a reflection coset; let $\left(d_{1}, \varepsilon_{1}\right), \ldots,\left(d_{n}, \varepsilon_{n}\right)$ be its generalized degrees (see for instance [2, 4.2]). For $\zeta$ a root of unity define $a(\zeta)$ as the multiset of the $d_{i}$ such that $\zeta^{d_{i}}=\varepsilon_{i}$. Then: 
(1) For any root of unity $\zeta$, the maximum dimension when w $\phi$ runs over $W \phi$ of a $\zeta$-eigenspace of $w \phi$ on $V \otimes_{k} k[\zeta]$ is $|a(\zeta)|$.

(2) For $w \phi \in W \phi$ denote $V_{w, \zeta} \subset V \otimes_{k} k[\zeta]$ its $\zeta$-eigenspace. Assume $\operatorname{dim} V_{w, \zeta}=|a(\zeta)|$ and let $C=C_{W}\left(V_{w, \zeta}\right)$ and $N=N_{W}\left(V_{w, \zeta}\right)$. Then $N / C$ is a complex reflection group acting on $V_{w, \zeta}$, with reflection degrees $a(\zeta)$.

(3) Any two subspaces $V_{w, \zeta}$ and $V_{w^{\prime}, \zeta}$ of dimension $|a(\zeta)|$ are $W$-conjugate.

(4) For $w \phi$ as in (2) the natural actions of $w \phi$ on $N$ and $C$ induce the trivial action on $N / C$.

(5) Let $a \in \mathbb{Z}$ be such that $(W \phi)^{a}=W \phi$ and such that $\zeta$ and $\zeta^{a}$ are conjugate by $\operatorname{Gal}(k[\zeta] / k)$. Then for $w \phi$ as in (2) there exists $v \in N_{W}(N) \cap N_{W}(C)$ which conjugates $w \phi C$ to $(w \phi)^{a} C$.

Proof. For (1) see for instance [2, 5.2], for (2) see [2, 5.6(3) and (4)] and for (3) see [2, 5.6 (1)]. (4) results from the observation that if $n \in N$ and $v \in V_{w, \zeta}$ then $\left(n^{-1} \cdot w_{n}\right)(v)=\left(n^{-1} w \phi n(w \phi)^{-1}\right)(v)=\left(n^{-1} w \phi n\right)\left(\zeta^{-1} v\right)=$ $\left(n^{-1} w \phi\right)\left(\zeta^{-1} n(v)\right)=\left(n^{-1}\right)(n(v))=v$ thus $n^{-1} \cdot w \phi_{n} \in C$.

For (5), $\operatorname{Gal}(k[\zeta] / k)$ acts naturally on $V \otimes_{k} k[\zeta]$, commuting with $G L(V)$, in particular with $W$ and $\phi$. If $\sigma \in \operatorname{Gal}(k[\zeta] / k)$ is such that $\sigma(\zeta)=\zeta^{a}$, let $\zeta^{a^{\prime}}=\sigma^{-1}(\zeta)$. Then $\sigma^{-1}\left(V_{w, \zeta}\right)=V_{w, \zeta^{a^{\prime}}}$. It follows that $N=N_{W}\left(V_{w, \zeta^{\prime}}\right)$ and $C=C_{W}\left(V_{w, \zeta^{a^{\prime}}}\right)$.

Now since $a^{\prime}$ is the inverse of $a$ modulo the order of $\zeta$ the space $V_{w, \zeta^{a^{\prime}}}$ is the $\zeta$-eigenspace of $(w \phi)^{a}$. By assumption we have $(w \phi)^{a} \in W \phi$. Since two maximal $\zeta$-eigenspaces of elements of $W \phi$ are conjugate by (3) there exists $v \in W$ which conjugates $V_{w, \zeta}$ to $V_{w, \zeta^{a^{\prime}}}$, and $v \in N_{W}(N) \cap N_{W}(C)$ since $N=N_{W}\left(V_{w, \zeta^{a^{\prime}}}\right)$ and $C=C_{W}\left(V_{w, \zeta^{a^{\prime}}}\right)$. The element $v$ thus conjugates the set $w \phi C$ of elements which have $V_{w, \zeta}$ as $\zeta$-eigenspace to the set $(w \phi)^{a} C$ of elements which have $V_{w, \zeta^{a^{\prime}}}$ as $\zeta$-eigenspace.

Generic Sylow subgroups. We define the Sylow $\Phi$-subtori of $(\mathbf{G}, F)$, first in the case when $\mathbf{G}$ is quasi-simple, then in the case of descent of scalars.

From now on we assume $\mathbf{G}$ semisimple. Then, if $\left(d_{1}, \varepsilon_{1}\right), \ldots,\left(d_{n}, \varepsilon_{n}\right)$ are the generalized degrees of the reflection coset $W \phi$, we have (see [9, 11.16])

$$
\left|\mathbf{G}^{F}\right|=q^{\sum_{i}\left(d_{i}-1\right)} \prod_{i}\left(q^{d_{i}}-\varepsilon_{i}\right)
$$


Proposition 2.10. Let $\mathbf{G}$ be as in Definition 1.1 and quasi-simple. Then we can rewrite the order formula (2.1) for $\left|\mathbf{G}^{F}\right|$ as

$$
\left|\mathbf{G}^{F}\right|=q^{\sum_{i}\left(d_{i}-1\right)} \prod_{\Phi \in \mathcal{P}} \Phi(q)^{n_{\Phi}}
$$

where $\mathcal{P}$ is a set of $q$-cyclotomic polynomials, and where $0 \neq n_{\Phi}=|a(\zeta)|$ (see Proposition 2.9) for any root $\zeta$ of $\Phi$. For each $\Phi \in \mathcal{P}$ there exists a non-trivial F-stable subtorus $\mathbf{S}_{\Phi}$ of $\mathbf{G}$ such that $\left|\mathbf{S}_{\Phi}^{F}\right|=\Phi(q)^{n_{\Phi}}$.

We note that if $\mathbf{G}^{F}$ is a Ree or Suzuki group, the $\eta$ of Definition 2.6 is 2. Otherwise $\eta=1$ and the $q$-cyclotomic polynomials are cyclotomic polynomials.

We call any $F$-stable torus $\mathbf{S}$ such that $\left|\mathbf{S}^{F}\right|$ is a power of $\Phi(q)$ a $\Phi$ torus, and tori $\mathbf{S}_{\Phi}$ as above are called Sylow $\Phi$-subtori of $(\mathbf{G}, F)$ - we abuse notation and call them Sylow $\Phi$-subtori of $\mathbf{G}$ when $F$ is clear from the context; they are the almost direct product of $n_{\phi} F$-indecomposable $\Phi$-tori.

Proof. Proposition 2.10 is essentially in [3] but let us reprove it.

First, we note that assuming $\left|\mathbf{G}^{F}\right|$ has a decomposition of the form (2.2), the value of $n_{\Phi}$ results from (2.1) : let $\zeta$ be any root of $\Phi(x)$. Then $(x-\zeta)$ divides $\Phi(x)$ with multiplicity one, and does not divide any another $\Phi^{\prime}(x)$ for $\Phi^{\prime} \in \mathcal{P}$ since the $\Phi(x / q)$ are distinct irreducible polynomials in $\mathbb{Q}[x]$. Thus $n_{\Phi}$ is the number of pairs $\left(d_{i}, \varepsilon_{i}\right)$ such that $x-\zeta$ divides $x^{d_{i}}-\varepsilon_{i}$.

There is a decomposition of the form (2.2): if $\eta=1$ we get such a decomposition of $\left|\mathbf{G}^{F}\right|$ by decomposing each term of (2.1) into a product of cyclotomic polynomials. Otherwise $\mathbf{G}^{F}$ is a Ree or Suzuki group, $\eta=2$ and $q$ is an odd power of $\sqrt{2}$ or $\sqrt{3}$, and the set $\mathcal{P}$ and the decomposition of the form (2.2) is given by what follows:

\begin{tabular}{c|cc}
$(\mathbf{G}, F)$ & $\left|\mathbf{G}^{F}\right|$ & generalized degrees of $W \phi$ \\
\hline${ }^{2} B_{2}\left(q^{2}\right)$ & $q^{4}\left(\Phi_{2,1} \Phi_{2,4}^{\prime} \Phi_{2,4}^{\prime \prime}\right)(q)$ & $\{(2,1),(4,-1)\}$ \\
${ }^{2} F_{4}\left(q^{2}\right)$ & $q^{24}\left(\Phi_{2,1}^{2} \Phi_{2,2}^{2} \Phi_{2,4}^{\prime 2} \Phi_{2,4}^{\prime \prime 2} \Phi_{2,6} \Phi_{2,12}^{\prime} \Phi_{2,12}^{\prime \prime}\right)(q)$ & $\{(2,1),(6,-1),(8,1),(12,-1)\}$ \\
${ }^{2} G_{2}\left(q^{2}\right)$ & $q^{6}\left(\Phi_{2,1} \Phi_{2,2} \Phi_{2,6}^{\prime} \Phi_{2,6}^{\prime \prime}\right)(q)$ & $\{(2,1),(6,-1)\}$
\end{tabular}

Note that for $\eta=2$ our " $q$-cyclotomic polynomials" are the " $(t p)$-cyclotomic polynomials" defined in [3, 3.14]. 
To construct the torus $\mathbf{S}_{\Phi}$ for $\Phi \in \mathcal{P}$, let us choose $\zeta$ a root of $\Phi$ and $w$ as in (2) of Proposition 2.9. Then if $\mathbf{T}_{w}$ is a maximal torus of type $w$ with respect to $\mathbf{T}$, so that $\left(\mathbf{T}_{w}, F\right) \simeq(\mathbf{T}, w F)$, the characteristic polynomial of $w \phi$ on $X(\mathbf{T})$ has $\Phi(x)^{n_{\Phi}}$ as a factor; the kernel of $\Phi(w \phi)$ on $X(\mathbf{T})$ is a pure sublattice corresponding to a subtorus $\mathbf{S}_{\Phi}$ of $\mathbf{T}_{w}$ such that $\left|\mathbf{S}_{\Phi}^{F}\right|=\Phi(q)^{n_{\Phi}}$.

Proposition 2.11. Let $(\mathbf{G}, F)$ be as in Definition 1.1, semisimple and such that the Dynkin diagram of $\mathbf{G}$ has $n$ connected components permuted transitively by $F$. Then there exists a reductive group $\mathbf{G}_{1}$ with isogeny $F_{1}$ such that up to isomorphism $\mathbf{G}$ is a "descent of scalars" $\mathbf{G}=\mathbf{G}_{1}^{n}$ with $F\left(g_{1}, \ldots, g_{n}\right)=\left(g_{2}, \ldots, g_{n}, F_{1}\left(g_{1}\right)\right)$.

Then $\mathbf{G}^{F} \simeq \mathbf{G}_{1}^{F_{1}}$, and if the scalar associated to $(\mathbf{G}, F)$ is $q$ that associated to $\left(\mathbf{G}_{1}, F_{1}\right)$ is $q_{1}:=q^{n}$. Thus we have $\left|\mathbf{G}^{F}\right|=q^{n \sum_{i}\left(d_{i}-1\right)} \prod_{\Phi \in \mathcal{P}} \Phi\left(q^{n}\right)^{n_{\Phi}}$ where $d_{i}, \mathcal{P}, n_{\phi}$ are as given by Proposition 2.10 for $\left(\mathbf{G}_{1}, F_{1}, q_{1}\right)$.

Here again, for $\Phi \in \mathcal{P}$ there exists a Sylow $\Phi$-subtorus of $\mathbf{G}$, that is an F-stable subtorus $\mathbf{S}_{\Phi}$ such that $\left|\mathbf{S}_{\Phi}^{F}\right|=\Phi\left(q^{n}\right)^{n_{\Phi}}$.

Proof. The proposition is obvious apart perhaps for the statement about the existence of $\mathbf{S}_{\Phi}$. This results in particular from the following lemma that we need for future reference.

Lemma 2.12. In the situation of Proposition 2.11, let $(\mathbf{T}, w F)$ where $\mathbf{T}=$ $\mathbf{T}_{1}^{n}$ be a maximal torus of type $w=\left(1, \ldots, 1, w_{1}\right)$ of $\mathbf{G}$ and define $\phi$ on $V=X(\mathbf{T}) \otimes \mathbb{C}\left(\right.$ resp. $\phi_{1}$ on $\left.V_{1}=X\left(\mathbf{T}_{1}\right) \otimes \mathbb{C}\right)$ by $F^{*}=q \phi\left(\right.$ resp. $\left.F_{1}^{*}=q_{1} \phi_{1}\right)$. Then if the characteristic polynomial of $w_{1} \phi_{1}$ is $P(x)$, that of $w \phi$ is $P\left(x^{n}\right)$. Let $\Phi$ be a $q_{1}$-cyclotomic factor of $P$ (corresponding to a $\mathbb{Z}[x]$-irreducible factor of the characteristic polynomial of $\left.w_{1} F_{1}^{*}\right)$ and let $\zeta$ be a root of $\Phi\left(x^{n}\right)$. Denote by $V_{\zeta}$ the $\zeta$-eigenspace of $w \phi$ (resp. by $V_{1, \zeta^{n}}$ the $\zeta^{n}$-eigenspace of $\left.w_{1} \phi_{1}\right)$.

Let $\mathbf{S}_{1}$ be the Sylow $\Phi$-subtorus of $\left(\mathbf{G}_{1}, F_{1}\right)$ determined by $\operatorname{Ker}\left(\Phi\left(w_{1} \phi_{1}\right)\right)$, and $\mathbf{S}$ be the $w F$-stable subtorus of $\mathbf{T}$ determined by $\operatorname{Ker}\left(\Phi\left((w \phi)^{n}\right)\right)$. Then $\mathbf{S}$ is a Sylow $\Phi$-subtorus of $(\mathbf{G}, F)$ and

$$
\frac{N_{W}\left(V_{\zeta}\right)}{C_{W}\left(V_{\zeta}\right)} \simeq \frac{N_{W_{1}}\left(V_{1, \zeta^{n}}\right)}{C_{W_{1}}\left(V_{1, \zeta^{n}}\right)} \simeq \frac{N_{\mathbf{G}_{1}}\left(\mathbf{S}_{1}\right)}{C_{\mathbf{G}_{1}}\left(\mathbf{S}_{1}\right)} \simeq \frac{N_{\mathbf{G}}(\mathbf{S})}{C_{\mathbf{G}}(\mathbf{S})}
$$

and we have an isomorphism $\mathbf{S}^{w F} \simeq \mathbf{S}_{1}^{w_{1} F_{1}}$ compatible with the actions of $N_{\mathbf{G}}(\mathbf{S}) / C_{\mathbf{G}}(\mathbf{S})$ and $N_{\mathbf{G}_{1}}\left(\mathbf{S}_{1}\right) / C_{\mathbf{G}_{1}}\left(\mathbf{S}_{1}\right)$ and the above isomorphism. 
Proof. Let $X=X(\mathbf{T}), X_{1}=X\left(\mathbf{T}_{1}\right)$. On $X \simeq X_{1}^{n}$ we have $F^{*}\left(x_{1}, \ldots, x_{n}\right)=$ $\left(x_{2}, \ldots, x_{n}, F_{1}^{*}\left(x_{1}\right)\right)$, thus $\phi\left(x_{1}, \ldots, x_{n}\right)=\left(q^{-1} x_{2}, \ldots, q^{-1} x_{n}, q_{1} q^{-1} x_{1}\right)$. It follows by an easy computation that $V_{\zeta}$ is equal to the set of $(x,(q \zeta) x, \ldots$, $\left.(q \zeta)^{n-1} x\right)$ where $x \in V_{1, \zeta^{n}}$, that $C_{W}\left(V_{\zeta}\right)=\left\{\left(v_{1}, \ldots, v_{n}\right) \mid v_{i} \in C_{W_{1}}\left(V_{1, \zeta^{n}}\right)\right\}$ and that $N_{W}\left(V_{\zeta}\right)=\left\{\left(v v_{1}, \ldots, v v_{n}\right) \mid v \in N_{W_{1}}\left(V_{1, \zeta^{n}}\right), v_{i} \in C_{W_{1}}\left(V_{1, \zeta^{n}}\right)\right\}$. This shows that $N_{W}\left(V_{\zeta}\right) / C_{W}\left(V_{\zeta}\right) \simeq N_{W_{1}}\left(V_{1, \zeta^{n}}\right) / C_{W_{1}}\left(V_{1, \zeta^{n}}\right)$. Since when $\zeta$ runs over the roots of $\Phi\left(x^{n}\right)$ the $q_{1} \zeta^{n}$ are roots of the same $\mathbb{Z}[x]$-irreducible polynomial $q_{1}^{\operatorname{deg} \Phi} \Phi\left(x / q_{1}\right)$, the $\zeta^{n}$ are Galois conjugate thus $C_{W_{1}}\left(V_{1, \zeta^{n}}\right)$ (resp. $\left.N_{W_{1}}\left(V_{1, \zeta^{n}}\right)\right)$ centralizes (resp. normalizes) all the conjugate eigenspaces, whence our claim that $N_{W_{1}}\left(V_{1, \zeta^{n}}\right) / C_{W_{1}}\left(V_{1, \zeta^{n}}\right) \simeq N_{\mathbf{G}_{1}}\left(\mathbf{S}_{1}\right) / C_{\mathbf{G}_{1}}\left(\mathbf{S}_{1}\right)$. Now $\operatorname{Ker}\left(\Phi\left((w \phi)^{n}\right)\right)$ is the span of $V_{\zeta}$ for all roots $\zeta$ of $\Phi\left(x^{n}\right)$ and by the analysis above $C_{W}\left(V_{\zeta}\right)$ and $N_{W}\left(V_{\zeta}\right)$ are independent of $\zeta$, thus isomorphic to $C_{W}(\mathbf{S})$ and $N_{W}(\mathbf{S})$.

We have the following commutative diagram

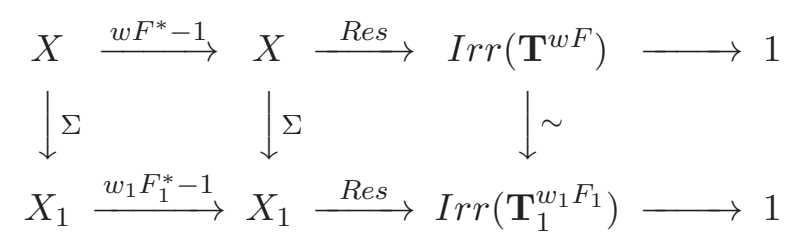

where $\Sigma$ is the map $\left(x_{1}, \ldots, x_{n}\right) \mapsto x_{1}+\ldots+x_{n}$. Since we have $\Sigma \circ$ $(w F)^{n}=w_{1} F_{1} \circ \Sigma$, for any polynomial $Q$ the morphism $\Sigma$ induces a surjective morphism $\operatorname{Ker}\left(Q\left(\left(w F^{*}\right)^{n}\right)\right) \rightarrow \operatorname{Ker}\left(Q\left(w_{1} F_{1}^{*}\right)\right)$ whence for $Q=P$ a surjection $\operatorname{Irr}\left(\mathbf{S}^{w F}\right) \rightarrow \operatorname{Irr}\left(\mathbf{S}_{1}^{w_{1} F_{1}}\right)$; since $\left|\mathbf{S}^{w F}\right|$ is prime to $\left|\mathbf{T}^{w F} / \mathbf{S}^{w F}\right|$ this surjection must be an isomorphism. Extended to $V=X \otimes \mathbb{C}$, the map $\Sigma$ sends $V_{\zeta}$ to $V_{1, \zeta^{n}}$ and sends the action of $N_{W}\left(V_{\zeta}\right) / C_{W}\left(V_{\zeta}\right)$ to that of $N_{W_{1}}\left(V_{1, \zeta^{n}}\right) / C_{W_{1}}\left(V_{1, \zeta^{n}}\right)$, whence the last statement of the lemma.

Note that any element of $W \phi$ is conjugate to an element of the form $\left(1, \ldots, 1, w_{1}\right) \phi_{1}$ so the form of $w$ in the statement of Lemma 2.12 covers all the types of maximal tori.

Remark 2.13. If the generalized degrees of $W_{1} \phi_{1}$ are $\left(d_{i}, \varepsilon_{i}\right)_{i}$ those of $W \phi$ are $\left(d_{i}, \eta_{i, j}\right)$ where $\eta_{i, j}$ for $j \in\{1, \ldots, n\}$ runs over the $n$-th roots of $\varepsilon_{i}$. It follows that $n_{\Phi}$ can be defined in terms of $W \phi$ as it is also the number of $\left(d_{i}, \eta_{i, j}\right)$ such that $\zeta^{d_{i}}=\eta_{i, j}$, where $\zeta$ is any root of $\Phi\left(x^{n}\right)$. 
Remark 2.14. For $\Phi \in \mathcal{P}(\mathbf{G})$, a Sylow $\Phi$-subtorus of $\mathbf{G}$ is a "power" of a subtorus $\mathbf{S}_{0}$ such that $\left|\mathbf{S}_{0}^{F}\right|=\Phi(q)$. If $\mathbf{G}$ is quasi-simple, such a subtorus $\mathbf{S}_{0}$ is $F$-indecomposable (since then the polynomial $\Phi$ is $q$-cyclotomic). But this is no longer true for a descent of scalars. First, a cyclotomic polynomial in $x^{n}$ decomposes in several cyclotomic polynomials according to the formula $\Phi_{d}\left(x^{n}\right)=\prod_{\left\{\mu \mid n, \frac{n}{\mu} \text { prime to } d\right\}} \Phi_{\mu d}(x)$ (see [3, Appendice 2]). But there could be further decompositions: for instance, the characteristic polynomial of $F^{*}$ on a Coxeter torus of a semisimple group $\mathbf{G}$ of type $B_{2}$ over $\mathbb{F}_{2}$ is $x^{2}+4$, which is $\mathbb{Z}$-irreducible. But on a descent of scalars $\mathbf{G} \times \mathbf{G}$, the characteristic polynomial of $F^{*}$ on a lift of scalars of this torus is $x^{4}+4$ which is no longer $\mathbb{Z}$-irreducible: $x^{4}+4=\left(x^{2}+2 x+2\right)\left(x^{2}-2 x+2\right)$, so the torus seen inside the descent of scalars is no longer $F$-indecomposable.

We could have decomposed $\left|\mathbf{G}^{F}\right|$ into a product of $q$-cyclotomic polynomials corresponding to $F$-indecomposable tori, but in the case of descent of scalars it was convenient to use larger tori.

Remark 2.15. An arbitrary semisimple reductive group is of the form $\mathbf{G}=$ $\mathbf{G}_{1} \ldots \mathbf{G}_{k}$, an almost direct product of descents of scalars of quasi-simple groups $\mathbf{G}_{i}$, corresponding to the orbits of $F$ on the connected components of the Dynkin diagram of $\mathbf{G}$. Then we have $\left|\mathbf{G}^{F}\right|=\left|\mathbf{G}_{1}^{F}\right| \cdots\left|\mathbf{G}_{k}^{F}\right|$ by Lemma 2.4 and similarly, if $\mathbf{S}$ is an $F$-stable torus of $\mathbf{G}$, and $\mathbf{S}_{i}=\mathbf{S} \cap \mathbf{G}_{i}$, then $\left|\mathbf{S}^{F}\right|=\left|\mathbf{S}_{1}^{F}\right| \ldots\left|\mathbf{S}_{k}^{F}\right|$. This can be used to give a global decomposition of $\left|\mathbf{G}^{F}\right|$, but the polynomials $\mathcal{P}$ in one factor could divide those in another. For instance we could have $\Phi_{2,4}^{\prime}$ for a factor of $\mathbf{G}$ of type ${ }^{2} B_{2}$ and $\Phi_{8}$ for another factor of type $B_{2}$. Because of this it is cumbersome to give a global statement.

From now on we fix $(\mathbf{G}, F)$ as in Proposition 2.11, which determines $q, n$, and $\eta$ minimal such that $q^{n \eta} \in \mathbb{Z}$. This allows in the next definition to omit the mention of $\mathbf{G}$ and $F$ from the notation $d(\ell)$.

Definition 2.16. Let $\ell$ be a prime number different from $p$. In the context of Proposition 2.11 we define $d(\ell)$ as the order of $q^{n \eta}(\bmod \ell)((\bmod 4)$ if $\ell=2)$.

In particular $\ell \mid \Phi_{d(\ell)}\left(q^{n \eta}\right)$. 
The next proposition extends some of the Sylow theorems of [3], and introduces a complex reflection group $W_{\Phi}$ attached to each $\Phi$ in the set $\mathcal{P}$ of Proposition 2.10.

Proposition 2.17. Under the assumptions of Proposition 2.11 , let $\mathbf{T}$ be an $F$-stable maximal torus of $\mathbf{G}$ in an F-stable Borel subgroup, and let $W \phi \subset$ $G L(X(\mathbf{T}))$ be the reflection coset associated to $(\mathbf{G}, F)$. Then for each $\Phi \in \mathcal{P}$ :

(1) If $\zeta$ is a root of $\Phi\left(x^{n}\right)$ and $w$ is as in Proposition 2.9(2), a maximal torus of $\mathbf{G}$ of type $w$ with respect to $\mathbf{T}$ contains a unique Sylow $\Phi$-subtorus $\mathbf{S}$.

For $\zeta, w$ as in (1) let $W_{\Phi}=N_{W}\left(V_{\zeta}\right) / C_{W}\left(V_{\zeta}\right)$ where $V_{\zeta}$ is the $\zeta$-eigenspace of $w \phi$ on $V=X(\mathbf{T}) \otimes \mathbb{C}$.

(2) For $\mathbf{S}$ as in (1) we have $N_{\mathbf{G}^{F}}(\mathbf{S}) / C_{\mathbf{G}^{F}}(\mathbf{S})=N_{\mathbf{G}}(\mathbf{S}) / C_{\mathbf{G}}(\mathbf{S}) \simeq W_{\Phi}$, and $W_{\Phi}$ can be identified to a subgroup of $G L(X(\mathbf{S}))$.

(3) The Sylow $\Phi$-tori of $\mathbf{G}$ are $\mathbf{G}^{F}$-conjugate.

(4) Let $\ell \neq p$ be a prime number, and assume that $\Phi$ divides $\Phi_{d(\ell)}$ (see Definition 2.16). Then unless $\ell=2$ and $\left(\mathbf{G}_{1}, F_{1}\right)$ is of type ${ }^{2} G_{2}$, any Sylow $\ell$-subgroup of $W_{\Phi}$ acts faithfully on the subgroup of $\ell$-elements $\mathbf{S}_{\ell}^{F}$ of $\mathbf{S}^{F}$.

Proof. For (1) we consider a torus $(\mathbf{T}, w F)$ of type $w$. Then a $w F$-stable subtorus corresponds to the span of a subset of eigenspaces of $w \phi$ on $V$. Since the polynomials $\Phi$ are prime to each other the polynomials $\Phi\left(x^{n}\right)$ are also, thus $q \zeta$ is root of no other factor of the characteristic polynomial of $w \phi$ than $\Phi\left(x^{n}\right)$. Thus the $\mathbf{S}$ defined in Lemma 2.12, which we will denote $\mathbf{S}_{0}$, is unique.

Let us show (2). Let $\left(\mathbf{T}_{w}, F, \mathbf{S}\right)$ be conjugate to $\left(\mathbf{T}, w F, \mathbf{S}_{0}\right)$. Let $\mathbf{L}=$ $C_{\mathbf{G}}(\mathbf{S})$, which, as the centralizer of a torus, is a Levi subgroup. Then we note that $N_{\mathbf{G}}(\mathbf{S}) \subset N_{\mathbf{G}}(\mathbf{L})$. It follows that we can find representatives of $N_{\mathbf{G}}(\mathbf{S})$ modulo $\mathbf{L}$ in $N_{\mathbf{G}}\left(\mathbf{T}_{w}\right)$ since for $n \in N_{\mathbf{G}}(\mathbf{S})$ the torus ${ }^{n} \mathbf{T}_{w}$ is another maximal torus of $\mathbf{L}$ which is thus $\mathbf{L}$-conjugate to $\mathbf{T}_{w}$. We thus get that $N_{\mathbf{G}}(\mathbf{S}) / \mathbf{L}=N_{\mathbf{G}}\left(\mathbf{S}, \mathbf{T}_{w}\right) /\left(N_{\mathbf{G}}\left(\mathbf{T}_{w}\right) \cap \mathbf{L}\right)$; transferring this to $\mathbf{T}$ and then to $W$ we get $N_{\mathbf{G}}\left(\mathbf{S}, \mathbf{T}_{w}\right) /\left(N_{\mathbf{G}}\left(\mathbf{T}_{w}\right) \cap \mathbf{L}\right) \simeq N_{W}\left(\mathbf{S}_{0}\right) / C_{W}\left(\mathbf{S}_{0}\right)$ where $\mathbf{S}_{0}$ is the subtorus of $\mathbf{T}$ determined by $\operatorname{Ker}\left(P\left(w F^{*}\right)\right)$ where $P=\Phi\left(x^{n} / q^{n}\right)$. The action of $F$ is transferred to the action of $w \phi$ on this quotient. 
That $N_{W}\left(\mathbf{S}_{0}\right)=N_{W}\left(V_{\zeta}\right)$ and $C_{W}\left(\mathbf{S}_{0}\right)=C_{W}\left(V_{\zeta}\right)$ was given in Lemma 2.12

By Proposition 2.9(4) we see that the action of $w \phi$ on $N_{W}\left(\mathbf{S}_{0}\right) / C_{W}\left(\mathbf{S}_{0}\right)$ is trivial, thus also that of $F$ on $N_{\mathbf{G}}(\mathbf{S}) / C_{\mathbf{G}}(\mathbf{S})$, thus $N_{\mathbf{G}}(\mathbf{S}) / C_{\mathbf{G}}(\mathbf{S})=$ $\left(N_{\mathbf{G}}(\mathbf{S}) / C_{\mathbf{G}}(\mathbf{S})\right)^{F}=N_{\mathbf{G}}(\mathbf{S})^{F} / C_{\mathbf{G}}(\mathbf{S})^{F}=N_{\mathbf{G}^{F}}(\mathbf{S}) / C_{\mathbf{G}^{F}}(\mathbf{S})$, the second equality since $\mathbf{L}=C_{\mathbf{G}}(\mathbf{S})$ is connected. Finally, the last part of (2) results from the fact that the representation of $W_{\Phi}$ on $X\left(\mathbf{S}_{0}\right)$, extended to $X\left(\mathbf{S}_{0}\right) \otimes \mathbb{C}$ has as summand the representation of $W_{\Phi}$ on $V_{\zeta}$, which is the reflection representation, thus faithful.

(3) is a direct translation of Proposition 2.9)(3): when brought to subtori of $\mathbf{T}$ corresponding to eigenspaces of $w \phi$ (resp. $w^{\prime} \phi$ ) the $\mathbf{G}^{F}$-conjugacy of two Sylow $\Phi$-subtori corresponds to the $W$-conjugacy of the corresponding eigenspaces.

For (4) we first remark that we can reduce to the case where $\mathbf{G}$ is quasisimple, using Lemma 2.12, Thus either $q \in \mathbb{Z}$ or $\mathbf{G}^{F}$ is a Ree or a Suzuki group. Let $\delta$ be the order of the coset $W \phi$, that is the smallest integer such that $(W \phi)^{\delta}=W$. We have $\delta \in\{1,2,3\}$. We first show the

Lemma 2.18. If $\mathbf{G}$ is quasi-simple and we are in one of the cases:

(1) $q \in \mathbb{Z}$ and $\delta \in\{1,2\}$.

(2) $q \in \mathbb{Z}, \delta=3$ and $d$ is prime to 3 .

(3) $q$ is an odd power of $\sqrt{2}$ and $\ell=3$.

then $W_{\Phi}$ acts faithfully on $\mathbf{S}_{\ell}^{F}$.

Proof. On $X(\mathbf{T}) \otimes \mathbb{Q}\left(q^{-1}\right)$ we have $w F^{*}=q w \phi$. The characteristic polynomial $Q$ of $w F^{*}$ on $X(\mathbf{S})$ is $q^{n_{\Phi} \operatorname{deg} \Phi} \Phi(x / q)^{n_{\Phi}}$; as $w F^{*}$ is semisimple, the minimal polynomial of $w F^{*}$ is $P=q^{\operatorname{deg} \Phi} \Phi(x / q)$. We can identify $X(\mathbf{S})$ with $\operatorname{Ker}(P(q w \phi))$ on $X(\mathbf{T})$. As in the proof of Proposition 2.8 , if $X=X(\mathbf{S})$ we can make $X^{\prime}=X \otimes \mathbb{Z}\left[q^{-1}\right]$ an $A$-module where $A=\mathbb{Z}\left[x, q^{-\eta}\right] / P$. Under the assumptions of the lemma $A$ is a Dedekind ring. This results from the proof of Proposition 2.8(1) when $q \in \mathbb{Z}$. In the remaining case (3) of Lemma 2.18, $\eta=2$ and the order of $q^{2}(\bmod 3)$ is 2 , thus $\Phi=x^{2}+1$ and $P=x^{2}+q^{2} ;$ we have $A=\mathbb{Z}\left[x, q^{-2}\right] / P \simeq \mathbb{Z}[1 / 2, \sqrt{-2}]$ which is integrally closed (thus Dedekind) since localized of $\mathbb{Z}[\sqrt{-2}]$ which is integrally closed. As an $A$-module of rank $n_{\Phi}$, the module $X^{\prime}$ is a sum of projective rank 1 
submodules thus $\mathbf{S}$ is a product of $n_{\Phi}$ copies of a $w F$-indecomposable torus. By Proposition 2.17(2) we can identify $W_{\Phi}$ to a subgroup of $G L(X)$. With the notations of Proposition 2.8, since the assumption of Proposition 2.8(1) is satisfied, $\bar{X}:=X /\left(w F^{*}-1\right) X \simeq \operatorname{Irr}\left(\mathbf{S}^{w F}\right)$ is isomorphic to $(\mathbb{Z} / \Phi(q))^{n_{\Phi}}$. The representation of $W_{\Phi}$ on $X$ reduces to $\bar{X}$. We will show it is faithful on $\bar{X} / \ell \bar{X}$ (or $\bar{X} / 4 \bar{X}$ when $\ell=2$ ).

If $q \in \mathbb{Z}$ and $\ell=2$ then $d \in\{1,2\}$ and we can apply Proposition [2.8(2) taking $m=4$. We get that $\bar{X} / 4 \bar{X} \simeq \operatorname{Ker}\left(w F^{*}-1 \mid X / 4 X\right)$. We have as observed in the proof of Proposition 2.8 that $\operatorname{Ker}\left(w F^{*}-1\right)=X / 4 X$ and the representation of $W_{\Phi}$ on $\bar{X} / 4 \bar{X}$, which is a quotient of $\operatorname{Irr}\left(\mathbf{S}_{\ell}^{w F}\right)$, is faithful by Lemma 4.3 .

If $q \in \mathbb{Z}$ and $\ell \neq 2$ then $d$ is prime to $\ell$; and in case (3) of Lemma $2.18 \eta=2, \ell=3$ thus $d=2$ and $\ell$ is prime to $d \eta$. In both cases we can apply Proposition 2.8(2) with $m=\ell$ to get that $\bar{X} / \ell \bar{X} \simeq \operatorname{Ker}\left(w F^{*}-1 \mid\right.$ $X / \ell X)$. We know by Lemma 4.3 that the representation of $W_{\Phi}$ on $X / \ell X$ is faithful and we would like to conclude that it is faithful on the submodule $\operatorname{Ker}\left(w F^{*}-1\right)$. We use the element $v$ given by Proposition 2.9)(5): it preserves the kernel of $\Phi(w \phi)$ thus induces an element of $G L(X)$ which defines an automorphism $\sigma$ of $W_{\Phi}$ which sends $w \phi$ to $(w \phi)^{a}$, so it remains true after reduction $(\bmod \ell)$ that $\sigma$ sends $w \phi$ to $(w \phi)^{a}$, thus permutes the eigenspaces of $w F^{*}$ on $X / \ell X$ : since $d$ is the order of $q(\bmod \ell)$, all the primitive $d$-th roots of unity live in $\mathbb{F}_{\ell}$ and the eigenvalues of $w F^{*}$ are the product of one primitive $d$-th root of unity, which is $q$, by the other primitive $d$-th roots of unity so are of the form $q^{1-a}$ where a runs over $(\mathbb{Z} / d)^{\times}$. And under the assumption $(W \phi)^{a}=W \phi$ of Proposition 2.9)(5) we can find $v$ thus $\sigma$ which sends the $q^{1-a}$-eigenspace of $w F^{*}$ to the $q^{1-1}=1$-eigenspace.

If every $a$ prime to $d$ has a representative in $1+\delta \mathbb{Z}$ we can satisfy $(W \phi)^{a}=W \phi$ for such $a$ thus every eigenspace is isomorphic as a $W_{\Phi}$-module to $\operatorname{Ker}\left(w F^{*}-1\right)$. Then $W_{\Phi}$ is faithful on the whole $X / \ell X$ if and only if it is faithful on $\operatorname{Ker}\left(w F^{*}-1\right)$, thus we conclude. If $a \equiv 1(\bmod \operatorname{gcd}(d, \delta))$ then by Bezout's theorem there exist integers $\alpha, \beta$ such that $a=1+\alpha d+\beta \delta$, and then $a-\alpha d \in 1+\delta \mathbb{Z}$ is a representative of $a$.

If $\delta=1$ or $\delta=2$ then every $a$ prime to $d$ is $\equiv 1(\bmod \operatorname{gcd}(d, \delta))$ and we conclude. We conclude similarly if $\delta=3$ and $d$ is prime to 3 , or in case (3) of Lemma 2.18 since in this case $d=2$. 
When $q \in \mathbb{Z}$ the only case not covered by the lemma is ${ }^{3} D_{4}$ and $d$ divisible by 3 , that is $d \in\{3,6,12\}$. But in this case $\ell>3$, since $d$ is the order of $q(\bmod \ell)$, thus $|W|$ is prime to $\ell$ and a fortiori the Sylow $\ell$-subgroup of $W_{\Phi}$ is trivial.

For the Ree and Suzuki groups we do not have to consider ${ }^{2} B_{2}$ since $W$ is a 2 -group and $\ell \neq p$, and the groups ${ }^{2} G_{2}$ since only the prime $\ell=2$ divides $|W|$ and is different from $p$, and this case is excluded in the proposition.

For the groups ${ }^{2} F_{4}$ the only prime $\ell \neq p$ such that $\ell|| W \mid$ is $\ell=3$ and we are in case $(3)$ of the lemma.

The Ree group ${ }^{2} G_{2}$ with $\ell=2$ is a genuine counterexample since the Sylow 2-subgroups of ${ }^{2} G_{2}(q)$ are isomorphic to $(\mathbb{Z} / 2)^{3}$.

\section{The Structure of the Sylow $\ell$-subgroups}

Definition 3.1. Let $\mathbf{G}, F, \mathbf{G}_{1}, \mathcal{P}$ and $n$ be as in Proposition 2.11 and let $\ell \neq p$ be a prime number. We define $D(\ell)$ as the set of integers $d$ such that for some $\Phi \in \mathcal{P}$ dividing $\Phi_{d}\left(x^{\eta}\right)$ we have $\ell \mid \Phi\left(q^{n}\right)$, where $\eta$ is as in Definition 2.16 .

The following proposition is [5, Théorème 1] when $\eta=1$; we give here a shorter proof. Since [5] was written, Malle ([7, 5.14 and 5.19]) has published a proof of (2) below — thus implicitly of (1) also - when $\eta=1$ (giving more, see Theorem 3.3).

Theorem 3.2. Assume in the situation of Definition 3.1 that $D(\ell) \neq \emptyset$, or equivalently that $\ell \| \mathbf{G}^{F} \mid$. Then

(1) $d(\ell) \in D(\ell)$.

(2) There exists a unique $\Phi \in \mathcal{P}$ such that $\ell \mid \Phi\left(q^{n}\right)$ and $\Phi$ divides $\Phi_{d(\ell)}\left(x^{\eta}\right)$. If $\mathbf{S}$ is a Sylow $\Phi$-torus then $N_{\mathbf{G}}(\mathbf{S})$ contains a Sylow $\ell$-subgroup of $\mathbf{G}^{F}$ which is an extension of $\left(Z^{0} C_{\mathbf{G}}(\mathbf{S})\right)_{\ell}^{F}$ by a Sylow $\ell$-subgroup of $W_{\Phi}$.

(3) The Sylow $\ell$-subgroups of $\mathbf{G}^{F}$ are abelian if and only if $|D(\ell)|=1$ (which is equivalent to $W_{\Phi}$ being an $\ell^{\prime}$-group), apart from the exception where $\left(\mathbf{G}_{1}, F_{1}\right)$ is of type ${ }^{2} G_{2}$ and $\ell=2$ in which case $|D(\ell)|=2$ and $\left|W_{\Phi}\right|=6$ but the 2-Sylow is abelian, isomorphic to $(\mathbb{Z} / 2)^{3}$.

Further, if $\mathbf{S}$ is as in (2), then $\left(Z^{0} C_{\mathbf{G}}(\mathbf{S})\right)_{\ell}^{F}=\mathbf{S}_{\ell}^{F}$ except if: 
- $\ell=3$ and $\mathbf{G}_{1}$ of type ${ }^{3} D_{4}$.

- $\ell=2, d=1$ and for some odd degree $\varepsilon_{i}=-1$. Equivalently $\mathbf{G}_{1}$ is nonsplit and has an odd reflection degree, that is, is one of ${ }^{2} A_{n},{ }^{2} D_{2 n+1}$ or ${ }^{2} E_{6}$.

- $\ell=2, d=2$ and for some odd degree $\varepsilon_{i}=1$; equivalently $\mathbf{G}_{1}$ is split and has an odd reflection degree, that is, is one of $A_{n}(n>1), D_{2 n+1}$ or $E_{6}$.

In the above exceptions, $Z^{0} C_{\mathbf{G}}(\mathbf{S})=C_{\mathbf{G}}(\mathbf{S})$ is a maximal torus of $\mathbf{G}$.

Proof. Let us note that to prove (2) when we are not in an exception, that is the stronger statement that a Sylow $\ell$-subgroup is in an extension of $\mathbf{S}^{F}$ by a Sylow $\ell$-subgroup of $W_{\Phi}$, it is enough to prove that

$$
v_{\ell}\left(\left|\mathbf{G}^{F}\right|\right)=v_{\ell}\left(\left|\mathbf{S}^{F}\right|\right)+v_{\ell}\left(\left|W_{\Phi}\right|\right)
$$

where $v_{\ell}$ denotes the $\ell$-adic valuation, and in the exceptions, if we have proved that $Z^{0} C_{\mathbf{G}}(\mathbf{S})=C_{\mathbf{G}}(\mathbf{S})$ it is enough to show

$$
v_{\ell}\left(\left|\mathbf{G}^{F}\right|\right)=v_{\ell}\left(\left|C_{\mathbf{G}}(\mathbf{S})^{F}\right|\right)+v_{\ell}\left(\left|W_{\Phi}\right|\right)
$$

Note also that by the definition of $d(\ell)$ and $D(\ell)$ in Proposition 2.11, assertion (1) as well as formulae (*) and (**) are equivalent in $\mathbf{G}$ and $\mathbf{G}_{1}$, that is we may assume $\mathbf{G}$ quasi-simple to prove them which we do now. Also, in view of (2) and Proposition $2.17(4)$, (3) reduces to proving:

$\left(3^{\prime}\right)|D(\ell)|=1$ is equivalent to $W_{\Phi}$ being an $\ell^{\prime}$-group.

We first look at the case of a Ree or Suzuki group, where $\eta=2$.

Let us prove (1) first. By Lemma 4.2 if $\ell$ divides $\left|\mathbf{G}^{F}\right|$ then there is an element of $D(\ell)$ of the form $d(\ell) \ell^{b}$ with $b \geq 0$. By inspecting the order formula for $\left|\mathbf{G}^{F}\right|$ given in the proof of Proposition 2.10 the elements of $D(\ell)$ have all their prime factors in $\{2,3\}$, so $b>0$ implies $\ell \in\{2,3\}$ thus $d(\ell) \in\{1,2\}$; inspecting again the formula, we see that then $d(\ell)$ in $D(\ell)$ and that $|D(\ell)|=1$ unless $\ell \in\{2,3\}$.

To prove (2) for $\ell \notin\{2,3\}$, we observe there is a single $\Phi \in \mathcal{P}$ such that $\ell \mid \Phi(q)$ since the two numbers $\Phi_{2,4}^{\prime}(q), \Phi_{2,4}^{\prime \prime}(q)$ are prime to each other, and the same observation applies to $\Phi_{2,6}^{\prime}(q), \Phi_{2,6}^{\prime \prime}(q)$ and $\Phi_{2,12}^{\prime}(q), \Phi_{2,12}^{\prime \prime}(q)$. Thus 
for $\ell \notin\{2,3\}$ assertions (3') and (*) are obvious since $\left|\mathbf{G}^{F}\right|_{\ell}=\left|\mathbf{S}^{F}\right|_{\ell}$ and $\ell \nmid|W|$.

Let us prove $(*)$ for $\ell \in\{2,3\}$; since $\ell \neq p$ and the elements of $D(\ell)$ have only 2 as prime factor in the case ${ }^{2} B_{2}$, we have just to consider:

- $\ell=3$ for ${ }^{2} F_{4}$ : we have $d(3)=2, W_{\Phi_{2,2}}=G_{12}$ of order 48 ; the only factor $\Phi(q)$ with a value divisible by 3 apart from $\left|\mathbf{S}^{F}\right|=\Phi_{2,2}(q)^{2}$ is $\Phi_{2,6}(q)$ and $v_{3}\left(\Phi_{2,6}(q)\right)=1=v_{3}\left(\left|G_{12}\right|\right)$ which proves this case.

- $\ell=2$ for ${ }^{2} G_{2}$ : we have $d(2)=2$ and $\left|W_{\Phi_{2,2}}\right|=6$; the only factor $\Phi(q)$ with an even value apart from $\left|\mathbf{S}^{F}\right|=\Phi_{2,2}(q)$ is $\Phi_{2,1}(q)$ and $v_{2}\left(\Phi_{2,1}(q)\right)=$ $1=v_{2}\left(\left|W_{\Phi}\right|\right)$ which proves this case.

We have seen (3') along the way.

Now we look at the other quasi-simple groups thus $\eta=1$. We notice generally that, assuming we have proved (1) then if $|D(\ell)|=1$ assertion (2) is trivial since a Sylow $\ell$-subgroup is then in $\mathbf{S}$, and (3') reduces to checking that $W_{\Phi}$ is an $\ell^{\prime}$-group.

We consider separately ${ }^{3} D_{4}$ where $\left|{ }^{3} D_{4}(q)\right|=q^{12}\left(\Phi_{1}^{2} \Phi_{2}^{2} \Phi_{3}^{2} \Phi_{6}^{2} \Phi_{12}\right)(q)$. Again, since the only prime factors of elements of $D(\ell)$ are $\{2,3\}$, we see that $d(\ell) \in D(\ell)$ except possibly if $\ell \in\{2,3\}$; but in that case $d(\ell) \in\{1,2\}$ and there is a factor $\Phi_{d(\ell)}(q)$, whence (1). Since $|W|=3 \cdot 2^{6}$ assertion (3') is proved when $D(\ell)=1$. It remains to prove $(2)$ when $\ell \in\{2,3\}$. In both cases $W_{\Phi_{d(\ell)}}=W\left(G_{2}\right)$ and by Lemma $4.2 v_{\ell}\left(\left|\mathbf{G}^{F}\right| /\left|\mathbf{S}^{F}\right|\right)=2$. If $\ell=2$ then $2=v_{\ell}\left(\left|W\left(G_{2}\right)\right|\right)$ which proves $(*)$. If $\ell=3$ a Sylow $\Phi$-subtorus $\mathbf{S}$ is in a torus $\mathbf{T}_{w}=C_{\mathbf{G}}(\mathbf{S})$ where $w=1$ if $d=1$ (resp. $w=w_{0}$ if $d=2$ ). We have $\left|\mathbf{T}_{1}^{F}\right|=\Phi_{1}(q)^{2} \Phi_{3}(q)$ (resp. $\left|\mathbf{T}_{w_{0}}^{F}\right|=\Phi_{2}(q)^{2} \Phi_{6}(q)$ ) which has same 3-valuation as $\left|\mathbf{G}^{F}\right| /\left|W_{\Phi}\right|$ which proves (**).

In the remaining cases $\varepsilon_{i}= \pm 1$ for all $i$. Let us set $\zeta_{d}=e^{2 i \pi / d}$. We have $\Phi=\Phi_{d(\ell)}$ and $v_{\ell}\left(\left|\mathbf{S}^{F}\right|\right)=\left|a\left(\zeta_{d(\ell))}\right)\right| v_{\ell}\left(\Phi_{d(\ell)}(q)\right)$.

We first treat the case $\ell$ odd. We have $a\left(\zeta_{d}\right)=\left\{d_{i} \mid \zeta_{d}^{d_{i}}=\varepsilon_{i}\right\}$ and $\left|W_{\Phi}\right|=\prod_{d_{i} \in a\left(\zeta_{d(\ell)}\right)} d_{i}$. By Lemma 4.2, a factor $\Phi_{e}(q)$ of $\left|\mathbf{G}^{F}\right|$ can contribute to the $\ell$-valuation only if $e$ is of the form $d(\ell) \ell^{b}$ for some $b \geq 0$. Further such a factor appears if and only if $a\left(\zeta_{e}\right) \neq \emptyset$, that is for some $i$ we have $\zeta_{d(\ell) \ell^{b}}^{d_{i}}=\varepsilon_{i}$. Since $\ell$ is odd raising this equality to the power $\ell^{b}$ gives $\zeta_{d(\ell)}^{d_{i}}=\varepsilon_{i}$ thus $d_{i} \in a\left(\zeta_{d(\ell)}\right)$ and in particular $d(\ell) \in D(\ell)$. And $\zeta_{d(\ell) \ell^{b}}^{d_{i}}=\varepsilon_{i}$ implies that $\ell^{b}$ divides $d_{i}$. Thus only the $d_{i}$ in $a\left(\zeta_{d(\ell)}\right)$ contribute to $v_{\ell}\left(\left|\mathbf{G}^{F}\right|\right)$ and each 
of them contributes $v_{\ell}\left(\Phi_{d(\ell)}(q)\right)+v_{\ell}\left(\Phi_{d(\ell) \ell}(q)\right)+\ldots+v_{\ell}\left(\Phi_{d(\ell) \ell_{\ell}\left(d_{i}\right)}(q)\right)$. By Lemma 4.2 this is $v_{\ell}\left(\Phi_{d(\ell)}(q)\right)+v_{\ell}\left(d_{i}\right)$. Summing over $d_{i} \in a\left(\zeta_{d(\ell)}\right)$ proves $(*)$.

It remains the case $\ell=2$ where we proceed similarly. We have $d(2) \in$ $\{1,2\}$. If $d(2)=1$ then $a(1)=\left\{d_{i} \mid \varepsilon_{i}=1\right\}$. Thus the condition $\zeta_{2^{b}}^{d_{i}}=\varepsilon_{i}$ is still equivalent to $2^{b} \mid d_{i}$; but there could be some more solutions of this equation than elements of $a(1)$ when $b=1$ : any odd $d_{i}$ such that $\varepsilon_{i}=-1$ brings an additional factor $1=v_{2}\left(\Phi_{2}(q)\right)$. If $d(2)=2$ then $a(-1)=\left\{d_{i} \mid\right.$ $\left.\varepsilon_{i}=(-1)^{d_{i}}\right\}$. The contribution of the even $d_{i}$ can be worked out as before; but this time the odd $d_{i}$ where $\varepsilon_{i}=1$ bring additional factors $v_{2}\left(\Phi_{1}(q)\right)$. In the exceptions in each case $C_{\mathbf{G}}(\mathbf{S})$ is a maximal torus of type 1 or $w_{0}$; looking at the orders of these tori, they contain enough extra $\Phi_{1}$ or $\Phi_{2}$ factors (which correspond to the eigenvalues 1 or -1 of $\phi$ or $w_{0} \phi$ ) to compensate the discrepancy.

Let us show now (3'), which reduces to proving that $|D(\ell)|>1$ implies $v_{\ell}\left(\left|W_{\Phi}\right|\right)>0$. Thus we assume $|D(\ell)|>1$. We first do the case $\ell=2$; then $d(\ell) \in\{1,2\}$ from which it follows, since the 1 and -1-eigenspaces are defined over the reals, that $W_{\Phi}$ is a Coxeter group, whose order is always even. We consider finally $\ell$ odd; then $D(\ell) \ni d(\ell)$ and $d(\ell) \ell^{a}$ for some $a>0$. But we have seen above that there exists a factor $\Phi_{d(\ell) \ell^{a}}(q)$ only if $\ell^{a} \mid d_{i}$ for some $d_{i}$ in $a\left(\zeta_{d(\ell)}\right)$.

We remark that if $\ell$ divides only one $\Phi_{d}(q)$, a Sylow $\ell$-subgroup $S$ lies in a single Sylow $\Phi$-torus $\mathbf{S}$ (the intersection of two tori has lower dimension so cannot have same order polynomial). It follows that $N_{\mathbf{G}^{F}}(S)=N_{\mathbf{G}^{F}}(\mathbf{S})$ and $C_{\mathbf{G}^{F}}(S)=C_{\mathbf{G}^{F}}(\mathbf{S})$. This observation is a start for describing the $\ell$-Frobenius category of $\mathbf{G}^{F}$ in terms of the category of $\zeta_{d}$-eigenspaces of $W_{\Phi_{d}}$.

In general, one can deduce the following unicity theorem from the work of Cabanes, Enguehard and Malle.

Theorem 3.3. Consider $\mathbf{G}, F, n, \mathbf{G}_{1}, q$ as in Proposition 2.11 with $q^{n} \in \mathbb{Z}$ and let $\Phi$ as defined in Theorem [3.2, (2). Assume that we are not in one of the following cases:

- $\ell=3, \mathbf{G}_{1}$ simply connected of type $A_{2},{ }^{2} A_{2}$ or $G_{2}$.

- $\ell=2, \mathbf{G}_{1}$ simply connected of type $C_{n}, n \geq 1$. 
Let $Q$ be a Sylow $\ell$-subgroup of $\mathbf{G}^{F}$. There is a unique Sylow $\Phi$-subtorus $\mathbf{S}$ of $\mathbf{G}$ such that $Q \subseteq N_{\mathbf{G}}(\mathbf{S})$.

Proof. In the context of Theorem 3.2(2), let $Q$ be a Sylow $\ell$-subgroup of $\mathbf{G}^{F}$ contained in $N_{\mathbf{G}}(\mathbf{S})$; then according to [4], $\mathbf{S}_{\ell}^{F}$ is often characteristic in $Q$ (for example when $l \geq 5$ ), thus in these cases $N_{\mathbf{G}^{F}}(Q) \subseteq N_{\mathbf{G}}\left(\mathbf{S}_{\ell}^{F}\right)$. Using inductively that property and inspecting small cases, G. Malle has proved the inclusion

$$
N_{\mathbf{G}^{F}}(Q) \subseteq N_{\mathbf{G}}(\mathbf{S})
$$

for all quasi-simple groups $\mathbf{G}$ short of the cases excluded in Theorem 3.3, see 7, Theorems 5.14 and 5.19]. Here $\mathbf{S}$ is a Sylow $\Phi_{d(\ell)}$-subtorus of $(\mathbf{G}, F)$ as defined in Definition 2.16 with $\eta=1$ (note that $N_{\mathbf{G}^{F}}(Q) \subseteq N_{\mathbf{G}}(\mathbf{S})$ implies $\left.Q \subseteq N_{\mathbf{G}}(\mathbf{S})\right)$.

We first verify that the last inclusion holds more generally in a "descent of scalars". With hypotheses and notations of Proposition 2.11 and Lemma 2.12 assume $q^{n} \in \mathbb{Z}$. If $e=d(\ell)$ is the order of $q^{n}$ modulo $\ell$, take $\Phi=\Phi_{e} \in \mathcal{P}$, defining $\mathbf{S}=\mathbf{S}_{\Phi}$ and $\mathbf{S}_{1}$. There is a morphism from $\mathbf{G}$ onto $\mathbf{G}_{1}$, sending $\mathbf{S}$ to $\mathbf{S}_{1}$, with restriction an isomorphism from $\mathbf{G}^{F}$ to $\mathbf{G}_{1}^{F}$. Then a Sylow -subgroup $Q_{1}$ of $\mathbf{G}_{1}^{F}$ contained in $N_{\mathbf{G}_{1}}\left(\mathbf{S}_{1}\right)$ is the isomorphic image of a Sylow $\ell$-subgroup $Q$ of $\mathbf{G}^{F}$ contained in $N_{\mathbf{G}}(\mathbf{S})$. The inclusion (3.1) written with $\left(\mathbf{G}_{1}, F_{1}, Q_{1}, \mathbf{S}_{1}\right)$ instead of $(\mathbf{G}, F, Q, \mathbf{S})$ implies (3.1) in $(\mathbf{G}, F)$.

From (3.1) the unicity of $\mathbf{S}$, given $Q$, follows:

Lemma 3.4. Let $\Phi \in \mathcal{P}$, let $\mathbf{S}$ be a Sylow $\Phi$-subtorus of $(\mathbf{G}, F)$ and $Q$ a Sylow $\ell$-subgroup of $\mathbf{G}^{F}$. If $N_{\mathbf{G}^{F}}(Q) \subseteq N_{\mathbf{G}}(\mathbf{S})$, then $\mathbf{S}$ is the unique Sylow $\Phi$-torus of $(\mathbf{G}, F)$ such that $Q \subseteq N_{\mathbf{G}}(\mathbf{S})$.

Proof. Assume $Q \subseteq N_{\mathbf{G}}\left(\mathbf{S}^{\prime}\right)$ for some Sylow $\Phi$-torus $\mathbf{S}^{\prime}$ of $(\mathbf{G}, F)$. By Proposition 2.17 there exists $g \in \mathbf{G}^{F}$ such that $\mathbf{S}=\left(\mathbf{S}^{\prime}\right)^{g}$, hence $Q^{g} \subseteq$ $N_{\mathbf{G}}(\mathbf{S})$. By Sylow's theorem in $N_{\mathbf{G}}(\mathbf{S})^{F}, Q=Q^{g h}$ for some $h \in N_{\mathbf{G}}(\mathbf{S})^{F}$ hence $g h \in N_{\mathbf{G}}(\mathbf{S})$ by our hypothesis.

\section{Appendix}

We gather here arithmetical lemmas used above. 
Lemma 4.1. Let $x, f, \ell \in \mathbb{N}$ where $\ell$ is prime, and assume $x \equiv 1(\bmod \ell)$ (resp. $(\bmod 4)$ if $\ell=2)$. Then $v_{\ell}\left(\frac{x^{f}-1}{x-1}\right)=v_{\ell}(f)$.

Proof. From $\frac{x^{f_{1} f_{2}-1}}{x-1}=\frac{x^{f_{1} f_{2}-1}}{x^{f_{2}-1}} \frac{x^{f_{2}-1}}{x-1}$ we see that it is enough to show the lemma when $f$ is prime. We have $\frac{x^{f}-1}{x-1}=f+\sum_{i=2}^{i=f}(x-1)^{i-1}\left(\begin{array}{l}f \\ i\end{array}\right)$. Let $S$ be this last sum; we have $S \equiv f(\bmod \ell)$, since $x-1 \equiv 0(\bmod \ell)$, thus $S$ is prime to $\ell$ when $f \neq \ell$ which shows the lemma in this case. When $f=\ell$ then all the terms of $S$ but the first one and possibly the last one are divisible by $\ell^{2}$ since $\left(\begin{array}{l}\ell \\ i\end{array}\right)$ is divisible by $\ell$ when $2 \leq i<\ell$; the last term is divisible by $\ell^{2}$ when $\ell-1 \geq 2$ which fails only for $f=\ell=2$; but when $\ell=2$ we have arranged that $v_{\ell}(x-1) \geq 2$ and this time $2(f-1) \geq 1$; thus $S \equiv f$ $(\bmod \ell)^{2}$, whence the lemma.

The following lemma is in [7, 5.2]; a short elementary proof results immediately from Lemma 4.1.

Lemma 4.2. Let $q, \ell \in \mathbb{N}$ where $\ell$ is prime. Let $d$ be the order of $q(\bmod \ell)$ (or $(\bmod 4)$ if $\ell=2)$. Then $\ell$ divides $\Phi_{e}(q)$ if and only if $e$ is of the form $d \ell^{b}$ with $b \in \mathbb{N}($ or additionally $b=-1$ when $\ell=d=2)$, and $v_{\ell}\left(\Phi_{d \ell^{b}}(q)\right)=1$ if $b \neq 0$.

The following lemma is in [8]; we give the proof since it is very short and the original German proof may be less accessible.

Lemma 4.3. Let $m \in \mathbb{N}, m>2$. Then the kernel of the reduction map $G L\left(\mathbb{Z}^{n}\right) \rightarrow G L\left((\mathbb{Z} / m)^{n}\right)$ is torsion-free.

Note that the bound $m>2$ is sharp since $-I d \equiv I d(\bmod 2)$.

Proof. Let $w \in G L\left(\mathbb{Z}^{n}\right)$ be of finite order, $w \neq I d$ and assume its reduction $v=I d$. We will derive a contradiction.

Possibly replacing $w$ by a power, we may assume that $w$ is of prime order $p$.

Also $G L\left(\mathbb{Z}^{n} / m\right)=\prod_{i} G L\left(\mathbb{Z}^{n} / p_{i}\right)$ where $m=\prod_{i} p_{i}$ is the decomposition of $m$ into prime powers, thus we may assume that $m$ is a prime power.

Since $w$ is of order $p$, the polynomial $\Phi_{p}(x)$ is a factor of the characteristic polynomial of $w$. The characteristic polynomial of $v$ is the reduction 
$(\bmod m)$ of that of $w$, thus we must have $\Phi_{p}(x)(\bmod m) \equiv(x-1)^{p-1} ;$ in particular $\left(\begin{array}{c}p-1 \\ 1\end{array}\right) \equiv-1(\bmod m)$ thus $m \mid p$ which implies $m=p$.

Write now $w=I d+x m^{a}$ where $x(\bmod m) \not \equiv 0$ and $a \in \mathbb{N}$. Then the equation $w^{m}=I d$ gives $\sum_{i=1}^{m}\left(\begin{array}{c}i \\ m\end{array}\right) x^{i} m^{a i}=0$, which after dividing by $m^{a+1}$ becomes $x=-\sum_{i=2}^{m}\left(\begin{array}{c}i \\ m\end{array}\right) x^{i} m^{a(i-1)-1}$ where all coefficients on the right-hand side are divisible by $m($ since $m \geq 3)$, which contradicts $x(\bmod m) \not \equiv 0$.

\section{References}

1. A. Borel, Linear Algebraic Groups, Springer GTM no. 126, 2nd ed. 1991.

2. M. Broué, Introduction to Complex Reflection Groups and Their Braid Groups, Lecture Notes in Mathematics 1988, Springer-Verlag, Berlin, 2010.

3. M. Broué and G. Malle, Théorèmes de Sylow génériques pour les groupes réductifs sur les corps finis, Math. Annalen, 292 (1992), 241-262.

4. M. Cabanes, Unicité du sous-groupe abélien distingué maximal dans certains sousgroupes de Sylow, C.R.A.S., 318 (1994), 889-894.

5. M. Enguehard, Sur les groupes de Sylow des groupes réductifs finis, unpublished notes of october 1992.

6. D. Gorenstein and R. Lyons, The local structure of finite groups of characteristic 2 type, Memoirs of AMS, 42, 1983.

7. G. Malle, Height 0 characters of finite groups of Lie type, Representation Theory 11(2007), 192-220.

8. H. Minkowski, Zur Theorie der positiven quadratischen Formen, J. Crelle, 101 (1887), 196-202.

9. R. Steinberg, Endomorphisms of linear algebraic groups, Memoirs of the A.M.S., 80 (1965). 